\title{
Navigating the New Landscape of Value-Based Care: An Example of Increasing Access, Improving Quality, and Reducing Costs Using the Unified Protocol
}

Matthew W. Southward1, Clair Cassiello-Robbins2, Rachel L. Zelkowitz3, \& M. Zachary

Rosenthal2

1Department of Psychology, University of Kentucky

2Department of Psychiatry \& Behavioral Sciences, Duke University Medical Center

3Department of Psychology and Human Development, Peabody College, Vanderbilt University

\section{Author Note}

Dr. Zelkowitz is now at Women's Health Science Division, National Center for PTSD, Veterans Affairs Boston Healthcare System.

Correspondence concerning this article should be addressed to Matthew W. Southward, University of Kentucky, 343 Waller Ave., Suite 303, Lexington, KY 40504. Phone: 859-562-

1569. Email: southward@uky.edu

The authors have no known conflicts of interests to disclose. The views expressed in this article are those of the authors and do not necessarily reflect the position or policy of the Department of Veterans Affairs or the United States government. 


\section{Navigating the New Landscape of Value-Based Care: An Example of Increasing Access, Improving Quality, and Reducing Costs Using the Unified Protocol}

In 2015, the U.S. Congress passed one of the largest reforms of federal healthcare payment policy since the creation of Medicare/Medicaid and the passage of the Affordable Care Act. MACRA (the Medicare Access and Children's Health Insurance Program Reauthorization Act of 2015) was designed to reimburse healthcare providers based on the value of their service, not the volume of patients treated. Termed value-based care, this policy aligns with a population health model focused on the "health outcomes of a group of individuals including the distribution of such outcomes within the group" (Kindig \& Stoddart, 2003). Starting in 2019, the law applied to clinical psychologists that met particular benchmarks based on service to Medicare patients.1

Value-based care is designed to shift financial risk from insurance payors to healthcare providers by financially incentivizing providers with upside (i.e., reward) and/or downside (i.e., penalty) contingencies linked to improved access to care and outcomes and reduced healthcare costs. Patient outcomes are weighed relative to the severity of presenting problems using the Centers for Medicaid and Medicare Services' hierarchically coded condition modifier so as not to disincentivize providers from treating patients with complex or severe presentations (Centers for Medicaid and Medicare Services, 2019). However, the increased uncertainty around yearly reimbursements may lead providers to treat fewer Medicare patients, leave the marketplace, or join larger practices. This shift in provider concentration could disproportionately impact areas with fewer behavioral health resources unless new providers or larger practices fill these gaps.

Given the influence of Medicare reimbursement policies on commercial insurance, value-

1 The law will apply to clinical psychologists, or their practice groups, who bill at least $\$ 90,000$ in a year to Medicare or who treat at least 200 patients with Medicare insurance. Providers who do not meet this Low Volume Threshold (LVT) may opt in to MACRA but will not be required to participate. 
based care models are expected to proliferate across the U.S. (Sharp et al., 2019). Clinical psychologists, especially those with training in (a) empirically supported, transdiagnostic, and process-based treatments (Hayes \& Hofmann, 2018), (b) research methods, and (c) program evaluation are well-suited to lead the implementation of behavioral interventions in the new era of value-based care. We will outline the three primary goals of value-based care and discuss the role of clinical psychologists in achieving these goals. We conclude by describing how the Unified Protocol for Transdiagnostic Treatment of Emotional Disorders (UP; Barlow et al., 2018) is a candidate treatment to implement in settings accountable to value-based incentives.

\section{Improving Behavioral Healthcare Access and Quality}

In contrast to traditional reimbursement models based on the frequency of patient visits, value-based care incentivizes providers to demonstrate the best outcomes for the lowest cost. It is not possible to accomplish this goal without improving access to healthcare services. Access can be defined by the population receiving necessary behavioral healthcare. For instance, although $56 \%$ of Americans require behavioral health services at some point in their life, $38 \%$ cannot access care due to barriers outside their control (e.g., transportation, long waitlists; Cohen Veterans Network, 2018). Even patients who do access care may be limited by the availability of evidence-based interventions (Gunter \& Whittal, 2010). Time-limited, evidence-based interventions may be one way to increase access. Although evidence-based behavioral health treatments are often brief (e.g., 12-16 sessions; American Psychological Association [APA], Division 12), patients typically attend fewer than five sessions (Minami et al., 2008). This discrepancy highlights the need for very brief treatments. One promising approach is modular treatments, consisting of individual skills that each achieve their intended effects (Chorpita et al., 2005). Although current implementations of modular treatments may not outperform more 
holistic treatments per-session (Barlow et al., 2017), individual skill modules may be more easily sequenced to maximize early treatment gains (Sauer-Zavala et al., 2019). Brief treatments can be further optimized by implementing homework assignments based on a thorough functional assessment of target symptoms or incorporating technology such as smartphone-supported apps.

\section{Enhancing Quality Through Integrated Care and Transdiagnostic Treatments}

High quality care is commonly operationalized and assessed by symptom improvement (Quality Payment Program).2 Clinical psychologists can confer value in this domain by providing leadership to systems about how to identify, routinely administer, and interpret the most appropriate outcome measures in interdisciplinary medical settings to demonstrate the quality of care. Given clinical psychologists' scientific training, they are expertly placed to provide, supervise, disseminate, and evaluate assessment strategies and identify evidence-based interventions to further increase quality in value-based care models.

Of course, clinical psychologists are not the only providers responsible for patients. In value-based care models, providers are incentivized to collaboratively treat patients' physical and behavioral health needs in an integrated care framework. In such models, providers may collaborate on treatment planning and outcome monitoring, using electronic medical records to implement protocols for stepped care. Integrated care models have been shown to be efficacious for certain disorders (e.g., depression) for nearly two decades (Unützer et al., 2002), and MACRA is expected to accelerate their proliferation. Providers can facilitate the use of integrated care by establishing typical care pathways for patients with particular clinical presentations. That is, each clinical presentation can be defined by a pathway to deliver evidence-based interventions known to change specific psychological mechanisms.

2 To incentivize providers to use electronic medical records, document patient progress in qualified clinical data registries, and avoid patient claims, these are also evaluated as indicators of care quality. 
One of the largest barriers to developing care pathways, however, is the gap between patients' common diagnostic presentations and the treatment research literature. Patients often present for treatment with a variety of comorbid diagnoses (Al-Asadi et al., 2015), but most evidence-based psychological treatments are designed to target specific disorders (APA, Division 12). These treatments may be ineffective for providers because they require expertise in a different treatment for each disorder, placing an unreasonable training burden on providers.

A parsimonious solution to this problem is to implement transdiagnostic treatments that target a range of disorders by intervening on the shared features thought to contribute to their etiology and maintenance. The development, implementation, and evaluation of such treatments represent important ways clinical psychologists may demonstrate leadership.

\section{Evidence-Based Psychological Interventions: Impact on Healthcare Costs?}

A primary goal of value-based care models is to reduce healthcare costs. A recent metaanalysis indicated that patients hospitalized for a somatic complaint with psychiatric comorbidity incurred higher medical costs, were more frequently re-hospitalized, and had longer lengths of stay than those without a psychiatric comorbidity (Jansen et al., 2018), suggesting effective treatment of psychiatric disorders could reduce system-wide healthcare costs. There is support for the greater cost-effectiveness of brief treatments, including cognitive-behavior therapy (CBT) for depression, relative to pharmacotherapy (Ross et al., 2019), and for similar levels of costeffectiveness relative to longer treatments for particular conditions and populations (e.g., Slade et al., 2017). Studies evaluating collaborative care models have also demonstrated costeffectiveness compared to usual care among patients with depressive (Jacob et al., 2012) and anxiety (Goorden et al., 2014) disorders. Given these results, it is reasonable to suggest that brief, transdiagnostic interventions could help reduce costs in value-based payor models. 


\section{An Example of Psychological Treatment in Value-Based Care}

To accomplish the primary goals of value-based care, behavioral health providers might consider care models based on treatments that target common mechanisms maintaining behavioral health disorders. These treatments could be flexible and modularized, using clinical research to guide their implementation. Evaluation of behavioral health interventions will require psychometrically valid, brief, easily accessible (e.g., free) measures of change (e.g., symptoms, functional impairment) that patients routinely complete. 3 Interventions and assessment measures that fit these criteria may best align with the contingencies of value-based care.

One transdiagnostic intervention that may confer value in the era of value-based care is the UP. The UP is a transdiagnostic cognitive-behavioral treatment for mood, anxiety, and related disorders. It is designed to facilitate an accepting and willing attitude toward the experience of strong emotions to reduce reliance on avoidance-based coping strategies. An accumulating body of evidence suggests the UP is efficacious for multiple clinical presentations, including anxiety, mood, obsessive-compulsive, and related disorders (Sakiris \& Berle, 2019).

Treatments such as the UP are well poised to meet the goals set forth by MACRA. The UP can increase patient access to care because it is a time-limited (8-16 session) protocol that consists of five core modules (understanding emotions, mindful emotion awareness, cognitive flexibility, countering emotional behaviors, interoceptive exposures). Preliminary data suggests these modules achieve their intended effects when delivered in isolation (Sauer-Zavala et al., 2017) and in a personalized order to match patients' strengths, which may lead to more rapid symptom improvement (Sauer-Zavala et al., 2019). Thus, even if patients only attend three to four sessions, they could still learn meaningful skills to improve their symptoms.

3 For instance: https://qpp.cms.gov/mips/explore-measures/quality-measures?py=2019\#measures and https://www.psychiatry.org/psychiatrists/practice/dsm/educational-resources/assessment-measures 
Standardized outcome monitoring is also embedded in the UP. Brief (five-item) assessments of functional impairment related to anxiety, depression, and "other" emotions (identified by the patient and therapist) are administered before each session and tracked throughout treatment. Because providers can directly compare patient progress on these metrics to the growing body of research on the efficacy of the UP (Sakiris \& Berle, 2019), the UP may be a good choice for providers who seek to demonstrate treatment quality.

Finally, the UP has the potential to be cost-effective. Although no researchers have evaluated its cost effectiveness to date, the UP has demonstrated efficacy across multiple primary disorders (Barlow et al., 2017) and comorbid conditions (Sauer-Zavala et al., 2020) comparable to single-disorder treatments, suggesting that it can be incorporated into care pathways for many diagnostic presentations. Providers could thus implement one treatment to (a) reduce training costs (McHugh \& Barlow, 2010), (b) save providers’ time preparing for sessions, and (c) potentially enhance provider competence in and fidelity to the treatment with repeated delivery. Investigation of the cost-effectiveness of the UP represents an important next step for the field.

\section{Future Directions}

The advantages of the UP in a value-based care model should be considered in light of its limitations. First, the standard form of the UP lasts 8-16 sessions, longer than many patients can complete (Minami et al., 2008). Ultra-brief versions of the UP may need to be adapted (Bentley et al., 2017) to enhance dissemination in community settings. Although ultra-brief transdiagnostic treatments may result in similar outcomes as more specialized or holistic treatments, providers may save on training costs by learning only one treatment. However, empirical data concerning the effectiveness of such dissemination efforts is currently lacking. Further, the UP has primarily been tested with anxiety or obsessive-compulsive disorders. 
Although these patients may comprise a large portion of cases (Delgadillo et al., 2014), especially among those who provide generalist care, comparisons of the UP to more holistic, evidence-based treatments for comorbid conditions is notably limited, albeit currently under investigation (Sauer-Zavala et al., 2020). Finally, the best method(s) for personalizing the UP (i.e., which skills, for whom, in what order; Sauer-Zavala et al., 2019) also requires further study. As mental health providers adjust to the realities of value-based care, the need for high quality, cost-effective interventions accessible to the population they serve will only increase. We believe the UP is an example of one potential intervention, although further rigorous studies of its economic effectiveness are needed. We encourage clinical psychologists to take a leading role in the changing landscape of healthcare by studying, developing, implementing, and supervising brief, modular, transdiagnostic interventions to improve population health. This may involve explicit graduate instruction in the economic and structural impact of healthcare policies, such as value-based care. It may also involve more emphasis in clinical training aligned with value-based models of real-world care, including (1) prioritizing direct patient care using brief, modular, transdiagnostic treatments, (2) providing more training in supervisory and administrative roles (e.g., learning about finance, management, etc.), (3) practicing program evaluation and quality assessment using clinical science methods and principles, (4) using novel approaches for brief and cost-effective behavior change that integrate digital health assessment and intervention, (5) training non-behavioral healthcare providers in strategies to manage behavioral health problems to offset medical costs, and (6) developing competence in wellness and prevention interventions. This breadth of training would equip new generations of clinical psychologists to apply their unique clinical and scientific talents to promote population health by increasing access to high-quality, evidence-based behavioral health interventions. 


\section{References}

Al-Asadi, A. M., Klein, B., \& Meyer, D. (2015). Multiple comorbidities of 21 psychological disorders and relationships with psychosocial variables: A study of online assessment and diagnostic system within a web-based population. Journal of Medical Internet Research, 17(2), e55. https://doi.org/10.2196/jmir.4143

American Psychological Association, Division 12. (2019). Research-supported psychological treatments. Retrieved from https://www.div12.org/psychological-treatments/

Barlow, D. H., Farchione, T. J., Bullis, J. R., Gallagher, M. W., Murray-Latin, H., Sauer-Zavala, S., Bentley, K. H., Thompson-Hollands, J., Conklin, L. R., Boswell, J. F., Ametaj, A., Carl, J. R., Boettcher, H. T., \& Cassiello-Robbins, C. (2017). The unified protocol for transdiagnostic treatment of emotional disorders compared with diagnosis-specific protocols for anxiety disorders: A randomized clinical trial. JAMA Psychiatry, 74(9), 875-884. https://doi.org/10.1001/jamapsychiatry.2017.2164

Barlow, D. H., Farchione, T. J., Sauer-Zavala, S., Murray Latin, H., Ellard, K. K., Bullis, J. R., \& Cassiello-Robbins, C. (2018). Unified protocol for transdiagnostic treatment of emotional disorders: Therapist guide. Oxford University Press.

Bentley, K. H., Sauer-Zavala, S., Cassiello-Robbins, C. F., Conklin, L. R., Vento, S., \& Homer, D. (2017). Treating suicidal thoughts and behaviors within an emotional disorders framework: Acceptability and feasibility of the unified protocol in an inpatient setting. Behavior Modification, 41(4), 529-557. https://doi.org/10.1177/0145445516689661

Centers for Medicaid and Medicare Services. (2019). 2019 merit-based incentive payment system complex patient bonus fact sheet. Department of Health and Human Services. Retrieved from https://qpp.cms.gov/about/resource-library 
Chorpita, B. F., Daleiden, E. L., \& Weisz, J. R. (2005). Modularity in the design and application of therapeutic interventions. Applied and Preventive Psychology, 11(3), 141-156. https://doi.org/10.1016/j.appsy.2005.05.002

Cohen Veterans Network. (2018). America’s Mental Health 2018. Retrieved from https://www.cohenveteransnetwork.org/wp-content/uploads/2018/10/Research-Summary10-10-2018.pdf

Delgadillo, J., McMillan, D., Leach, C., Lucock, M., Gilbody, S., \& Wood, N. (2012). Benchmarking routine psychological services: A discussion of challenges and methods. Behavioural and Cognitive Psychotherapy, 42(1), 16-30. https://doi.org/10.1017/S135246581200080X

Goorden, M., Muntingh, A., van Marwijk, H., Spinhoven, P., Adèr, H., van Balkom, A., van der Feltz-Cornelis, C., \& Hakkaart-van Roijen, L. (2014). Cost utility analysis of a collaborative stepped care intervention for panic and generalized anxiety disorders in primary care. Journal of Psychosomatic Research, 77(1), 57-63. https://doi.org/10.1016/j.jpsychores.2014.04.005

Gunter, R. W., \& Whittal, M. L. (2010). Dissemination of cognitive-behavioral treatments for anxiety disorders: Overcoming barriers and improving patient access. Clinical Psychology Review, 30(2), 194-202. https://doi.org/10.1016/j.cpr.2009.11.001

Hayes, S. C., \& Hofmann, S. G. (Eds.) (2018). Process-based CBT: The science and core clinical competencies of cognitive behavioral therapy. New Harbinger.

Jacob, V., Chattopadhyay, S. K., Sipe, T. A., Thota, A. B., Byard, G. J., \& Chapman, D. P. (2012). Economics of collaborative care for management of depressive disorders: A community guide systematic review. American Journal of Preventive Medicine, 42(5), 
539-549. https://doi.org/10.1016/j.amepre.2012.01.011

Jansen, L., van Schijndel, M., van Waarde, J., \& van Busschbach, J. (2018). Health-economic outcomes in hospital patients with medical-psychiatric comorbidity: A systematic review and meta-analysis. PLoS ONE, 13, e0194029. https://doi.org/10.1371/journal.pone.0194029

Kindig, D., \& Stoddart, G. (2003). What is population health? American Journal of Public Health, 93(3), 380-383. https://doi.org/10.2105/ajph.93.3.380

McHugh, R. K., \& Barlow, D. H. (2010). The dissemination and implementation of evidencebased psychological treatments. American Psychologist, 65(2), 73-84. https://doi.org/10.1037/a0018121

Medicare Access and CHIP Reauthorization Act of 2015, Pub. L. No. 114-10, Stat. 87 (2015).

Minami, T., Wampold, B. E., Serlin, R. C., Hamilton, E. G., Brown, G. S., \& Kircher, J. C. (2008). Benchmarking the effectiveness of psychotherapy treatment for adult depression in a managed care environment: A preliminary study. Journal of Consulting and Clinical Psychology, 76(1), 116-124. https://doi.org/10.1037/0022-006X.76.1.116

Quality Payment Program. Retrieved December 18, 2019 from https://qpp.cms.gov

Ross, E. L., Vijan, S., Miller, E. M., Valenstein, M., \& Zivin, K. (2019). The cost-effectiveness of cognitive-behavior therapy versus second-generation antidepressants for initial treatment of major depressive disorder in the United States: A decision analytic model. Annals of Internal Medicine, 171(11), 785-795. https://doi.org/10.7326/M18-1480

Sakiris, N., \& Berle, D. (2019). A systematic review and meta-analysis of the Unified Protocol as a transdiagnostic emotion regulation based intervention. Clinical Psychology Review, 72, 101751. https://doi.org/10.1016/j.cpr.2019.101751 
Sauer-Zavala, S., Bentley, K. H., Jarvi Steele, S., Wilner Tirpak, J., Ametaj, A. A., Nauphal, M., Cardona, N., Wang, M., Farchione, T. J., \& Barlow, D. H. (2020). Treating depressive disorders with the unified protocol: A preliminary randomized evaluation. Journal of Affective Disorders, 264, 438-445. https://doi.org/10.1016/j.jad.2019.11.072

Sauer-Zavala, S., Cassiello-Robbins, C., Ametaj, A. A., Wilner, J. G., \& Pagan, D. (2019).

Transdiagnostic treatment personalization: The feasibility of ordering unified protocol modules according to patient strengths and weaknesses. Behavior Modification, 43(4), 518-543. https://doi.org/10.1177\%2F0145445518774914

Sauer-Zavala, S., Cassiello-Robbins, C., Conklin, L. R., Bullis, J. R., Thompson-Hollands, J., \& Kennedy, K. A. (2017). Isolating the unique effects of the Unified Protocol treatment modules using single case experimental design. Behavior Modification, 41(2), 286-307. https://doi.org/10.1177/0145445516673827

Sharp, J. P., Conway, P. H., \& Rajkumar, R. (2019). Engineering a rapid shift to value-based payment in North Carolina: Goals and challenges for a commercial ACO program. NEJM Catalyst. Retrieved from https://catalyst.nejm.org/blue-premier-nc-value-based-payment/

Slade, E. P., Gottlieb, J. D., Lu, W., Yanos, P. T., Rosenberg, S., Silverstein, S. M., Minsky, S. K., \& Mueser, K. T. (2017). Cost-effectiveness of a PTSD intervention tailored for individuals with severe mental illness. Psychiatric Services, 68(12), 1225-1231. https://doi.org/10.1176/appi.ps.201600474

Unützer, J., Katon, W., Callahan, C. M., Williams, J. W., Hunkeler, E., Harpole, L., ... Langston, C. (2002). Collaborative care management of late-life depression in the primary care setting: A randomized controlled trial. JAMA Psychiatry, 288(22), 2836-2845. https://doi.org/10.1001/jama.288.22.2836 